\title{
Solving lorenz system of equation by Laplace homotopy analysis method
}

\author{
G. Sai Sundara Krishnan ${ }^{1}$, R.Malathy ${ }^{2}$, S.R.Saratha ${ }^{3}$ \\ \{ ssk.amcs@psgtech.ac.in ${ }^{1}$, jaysaran88@gmail.com², ammusaratha@gmail.com ${ }^{3}$ \} \\ Applied Mathematics and Computational Sciences, PSG College of Technology, Coimbatore, India ${ }^{1}$, \\ Department of Mathematics, SNS College of Engineering, Coimbatore, India ${ }^{2}$, Department of \\ Mathematics, Kumaraguru College of Technology, Coimbatore, India ${ }^{3}$
}

\begin{abstract}
The Laplace Homotopy Analysis Method Via Modified Riemann-Liouville Integral has been explored from a new perspective. The important items are assimilated, and the result is proven using well-defined evidence. A combination of the homotopy analysis approach and the suggested integral transform is used to find fractional differential equations
\end{abstract}

Keywords: Homotopy, Homotopy Analysis Method, Laplace Transform, Fractional Differential Equations.

\section{Introduction}

Many researchers have recently become interested in the theoretical studies of various integral transforms as a methodical mathematical strategy for converting fractional differential equations into simple algebraic formulas. Chowdhury et al (2007) introduced MHPM to handle the Lorenz system in the literature. The DTM was used by Mossa et al (2008) to solve a non-linear differential equation.

Jumarie (2009) presented a fractional-order Laplace's transform definition for functions that are fractionally differentiable but not differentiable. Alomari et al (2011) modified the DTM to achieve continuous and analytic solutions for each interval when solving non-linear fractional differential equations. The Laplace transform was first introduced to the field by Liang et al (2015).Medina et al (2017) an investigated the effect of the fractional Laplace Transform incorporated in the RiemannLiouville Fractional Derivative. For various fractional linear differential equations with constant coefficients, Silva et al (2018) studied the pleasant fractional derivative. The integral trans-form is used in conjunction with the Homotopy Analysis Method to solve nonlinear differential equations (HAM). Hariharan (2017) proposed the homotopy analysis technique (HAM) for solving a few partial differential equations in the literature. Mohammed et al (2017) used modified Laplace Homotopy Analysis to solve a nonlinear system of fractional partial differential equations.

The Fractional Laplace Transform through Modified Riemann-Liouville derivative [Jumarie (2009)] is the subject of this work. It is coupled with HAM, which was proposed by Liao (1992), to create a novel hybrid approach called Laplace Homotopy Analysis

through Modified RiemannnLiouville Integral.Nonlinear fractional differential equations can be solved using this hybrid approach. 
Edward Lorenz was the first to study the Lorenz system, which is a set of ordinary differential equations. For specific parameter values and starting circumstances, it is renowned for having chaotic solutions. The Lorenz attractor, in particular, is a collection of chaotic Lorenz system solutions.

This research motivates to study the Fractional Laplace transform via Modified RiemannLiouville [Jumarie (2009)] and it is combined with HAM introduced by Liao (1992), which provides the new hybrid technique Laplace Homotopy Analysis method via Modified Riemannn-Liouville Integral.

Non-chaotic behaviour is obtained in this work by employing modal series solutions with Rayleigh number $\mathrm{R}$ parameter values below the critical value. Throughout this study, we assume $\sigma=10, \mathrm{~b}=-8 / 3$, and change the Rayleigh number $\mathrm{R}$ to get different dynamical behaviours and assess the recommended approach. Pandemonium is well known to occur around the critical parameter value $R=24.74$. [10]

\section{Preliminaries}

This part contains the essential definitions for the study, as well as other fundamental results that can be found in Jumarie (2009).

Definition 2.1: The Mittag-Leffler fun€€ction which is a generalization of exponential function is defined as

$$
E_{\zeta}(z)=\sum_{n=0}^{\infty} \frac{z^{n}}{\Gamma(\zeta n+1)}
$$

Where $\zeta \in \mathrm{C}, \mathrm{R}(\zeta)>0$.

Definition 2.2: The continuous function $g: R \rightarrow R, \quad t \rightarrow g(t)$ has a fractional derivative of order $k \zeta$. For any positive integer $\mathrm{k}$ and for any $\zeta, 0<\zeta \leq 1$, the Taylor's series of

Fractional order can be expressed as

$$
g(t+h)=\sum_{k=0}^{\infty} \frac{h^{\zeta k}}{(\zeta k) !} g^{(\zeta k)}(t), 0<\zeta \leq 1
$$

Where $\Gamma(1+\zeta \mathrm{k})=(\zeta \mathrm{k})$ !.

Definition 2.3: (Modified Riemann Liouville derivative) Let $\quad q: R \rightarrow R$, denote a continuous (but not necessarily differentiable) function.

i. Assume that $q(y)$ is a constant $K$. Then its fractional derivative of order $\zeta$ is

$$
D_{y}^{\zeta}=K \Gamma^{-1}(1-\zeta) y^{-\zeta}, \zeta \leq 0 \text {, }
$$

ii. When $q(y)$ is not a constant, then we will set

$$
q(y)=q(0)+(q(y)-q(0)),(3)
$$

in which, for negative $\zeta$, one has

$$
D_{y}^{\zeta}(q(y)-q(0))=D_{y}^{\zeta} q(y)=D_{y}\left(q^{(\zeta-1)}(y)\right) \text {. }
$$

(4) When $n \leqslant \zeta<n+1$, we will set

$$
q^{(\zeta)}(y)=\left(q^{(\zeta-n)}(y)\right)^{(n)}, n \geqslant 1 \text {. }
$$

In order to find the fractional derivative of compound functions, equation (6) is used. 


$$
\begin{aligned}
& d^{\zeta} q \cong \Gamma(1+\zeta) d q, 0<\zeta<1 . \\
& \text { Definition } 2.4: \text { If } 0<\zeta<1 \text { then } \\
& D_{y}^{\zeta} y^{\eta}=\Gamma(\eta+1) \Gamma^{-1}(\eta+1-\zeta) y^{\eta-\zeta}, \eta>0, \\
& \text { Or, if } \zeta=n+\theta, n \in \mathbb{N}, \text { then } \\
& D_{y}^{n+\theta} y^{\eta}=\Gamma(\eta+1) \Gamma^{-1}(\eta+1-n-\theta) y^{\eta-n-\theta}, 0<\theta<1, \text { (8) }
\end{aligned}
$$

\section{Fractional Laplace Transform Via Modified Riemann-Liouville Integral}

Definition 3.1: Let $k(y)$ denote a function that vanishes for the negative value of $\mathrm{y}$. Its Laplace's transformation $L_{\zeta} k(y)$ of order $\zeta$ defined by the following expression, where it is finite:

$$
\begin{aligned}
& L_{\zeta}[k(y)]=K_{\zeta}(s)=\int_{0}^{\infty} E_{\zeta}\left[-s^{\zeta} y^{\zeta}\right] k(y)(d y)^{\zeta}, \\
= & \lim _{M \rightarrow \infty} \int_{0}^{M} E \zeta\left[-s^{\zeta} y^{\zeta}\right] k(y)(d y)^{\zeta}
\end{aligned}
$$

where $s \in C$, and $E_{\zeta}$ is the Mittag-Leffler function $\sum u^{k} /(k \zeta)$ !.

Theorem 3.1: if $L_{\zeta}[k(y)]=K_{\zeta}(s)$ then

Scaling property

(i) $L_{\zeta}[k(\text { ay })]_{s}=\frac{1 \zeta}{a} L_{\zeta}[k(y)]_{\frac{s}{a}}$,

Shifting Property

$$
\text { (ii) } L_{\zeta}[k(y-b)]=E_{\zeta}\left(-s^{\zeta} b^{\zeta}\right) L_{\zeta}[k(y)] \text {, }
$$

Frequency Shifting Property

$$
(\text { iii }) L_{\zeta}\left[E_{\zeta}\left(-c^{\zeta} y^{\zeta}\right) k(y)\right]_{s}=L_{\zeta}[k(y)]_{s+c},(13)
$$

Derivative Property

$$
\text { (iv) } L_{\zeta}\left[-y^{\zeta} k(y)\right]_{s}=D_{s}^{\zeta} L_{\zeta}[k(y)],(14)
$$

Laplace transform of fractional derivative

$$
(v) L_{\zeta}\left[k^{\zeta}(y)\right]=s_{\zeta} L_{\zeta}[k(y)]-\Gamma(1+\zeta) g(0) \text {. }
$$

Theorem 3.2: Let the convolution of the two functions $\mathrm{k}(\mathrm{y})$ and $\mathrm{l}(\mathrm{y})$ of order $\zeta$ is given by

$(k(y) * l(y))_{\zeta}=\int_{0}^{y} k(y-u) l(y)(d u)^{\zeta}$,

then one has the equality

$$
L_{\zeta}\left[(k(y) * l(y))_{\zeta}\right]=L_{\zeta}[k(y)] L_{\zeta}[l(y)] .
$$

Coming up next are the main ends for the Laplace fractional change of standard functions, as displayed in Table 1:

\begin{tabular}{|l|l|c|}
\hline \multicolumn{3}{|c|}{ Laplace Fractional transform of standard functions } \\
\hline S.N & $\mathrm{g}(\mathrm{y})$ & $L_{\zeta}[g(y)]=G_{\zeta}(s)$ \\
\hline 1 & 1 & $\frac{1}{s^{\zeta}} \Gamma(\zeta+1)$ \\
\hline
\end{tabular}




\begin{tabular}{|c|c|c|}
\hline 2 & $t^{\zeta}$ & $\frac{1}{s^{2 \zeta}} \Gamma^{2}(\zeta+1)$ \\
\hline 3 & $t^{2 \zeta}$ & $\frac{1}{s^{3 \zeta}} \Gamma^{3}(\zeta+1) \Gamma(3)$ \\
\hline 4 & $t^{n \zeta}$ & $\begin{array}{r}\frac{1}{s^{(n+1) \zeta}} \Gamma^{n+1}(\zeta+1) \Gamma(n \\
+1)\end{array}$ \\
\hline 5 & $E_{\zeta}\left[a^{\zeta} y^{\zeta}\right]$ & $\frac{1}{(s-a)^{\zeta}} \Gamma(\zeta+1)$ \\
\hline 6 & $E_{\zeta}\left[-a^{\zeta} y^{\zeta}\right]$ & $\frac{1}{(s+a)^{\zeta}} \Gamma(\zeta+1)$ \\
\hline 7 & $E_{\zeta}\left[i^{\zeta} a^{\zeta} y^{\zeta}\right]$ & $\frac{1}{(s-i a)^{\zeta}} \Gamma(\zeta+1)$ \\
\hline 8 & $E_{\zeta}\left[-i^{\zeta} a^{\zeta} y^{\zeta}\right]$ & $\frac{1}{(s+i a)^{\zeta}} \Gamma(\zeta+1)$ \\
\hline 9 & $\sin \left(a^{6} y^{6}\right)$ & $\frac{a^{\zeta}}{\left(s^{2}+a^{2}\right)^{\zeta}} \Gamma(\zeta+1)$ \\
\hline 10 & $\cos \left(a^{\zeta} y^{\zeta}\right)$ & $\frac{s^{\zeta}}{\left(s^{2}+a^{2}\right)^{\zeta}} \Gamma(\zeta+1)$ \\
\hline 11 & $\sinh \left(a^{\zeta} y^{6}\right)$ & $\frac{a^{\zeta}}{\left(s^{2}-a^{2}\right)^{\zeta}} \Gamma(\zeta+1)$ \\
\hline 12 & $\cosh \left(a^{\zeta} y^{\zeta}\right)$ & $\frac{s^{\zeta}}{\left(s^{2}-a^{2}\right)^{\zeta}} \Gamma(\zeta+1)$ \\
\hline 13 & $E_{\zeta}\left[a^{\zeta} y^{\zeta}\right] \sin \left[b^{\zeta} y^{\zeta}\right]$ & $\begin{array}{r}\frac{b^{\zeta}}{\left(s^{\zeta}-a^{\zeta}\right)^{2 \zeta}+b^{2 \zeta}} \Gamma(\zeta \\
+1)\end{array}$ \\
\hline 14 & $E_{\zeta}\left[a^{\zeta} y^{\zeta}\right] \cos \left[b^{\zeta} y^{\zeta}\right]$ & $\begin{array}{r}\frac{(s-a)^{\zeta}}{\left(s^{\zeta}-a^{\zeta}\right)^{2 \zeta}+b^{2 \zeta}} \Gamma(\zeta \\
+1)\end{array}$ \\
\hline 15 & $E_{\zeta}\left[a^{\zeta} y^{\zeta}\right] \sinh \left[b^{\zeta} y^{\zeta}\right]$ & $\begin{array}{r}\frac{b^{\zeta}}{\left(s^{\zeta}-a^{\zeta}\right)^{2 \zeta}-b^{2 \zeta}} \Gamma(\zeta \\
+1)\end{array}$ \\
\hline 16 & $E_{\zeta}\left[a^{\zeta} y^{\zeta}\right] \cos \left[b^{\zeta} y^{\zeta}\right]$ & $\begin{array}{r}\frac{(s-a)^{\zeta}}{\left(s^{\zeta}-a^{\zeta}\right)^{2 \zeta}-b^{2 \zeta}} \Gamma(\zeta \\
+1)\end{array}$ \\
\hline
\end{tabular}

The Laplace transform will be utilized related to the

fractional homotopy analysis way to deal with tackle both linear and nonlinear differential conditions (Fractional Homotopy Analysis method).

\section{Fractional laplace homotopy analysis}

METHOD (FLHAM) 
Consider the fractional time nonlinear differential condition with the accompanying beginning condition:

$D^{\zeta} v(y, t)+R(y, t)+N v(y, t)=q(y, t), v(y, 0)=k(y),(18)$

where $D^{\zeta}$ is the fractional differential operator $\quad D^{\zeta}=\frac{\partial^{\zeta}}{\partial t \zeta} \mathrm{R}$ is the differential linear operator, $\mathrm{N}$ is the differential non-linear operator and $\mathrm{q}(\mathrm{y} ; \mathrm{t})$ is source term.

To solve the non-linear partial differential condition,

Embrace the accompanying organized method:

Step 1: Apply fractional laplace transform, the equation (18),

$\left[D^{\zeta} v(y, t)\right]+L_{\zeta}[R v(y, t)]+L_{\zeta}[N v(y, t)]=L_{\zeta}[q(y, t)] . \quad$ (19)

Step 2: Applying the derivative of fractional Laplace transform,

The condition (19), can communicate as

$L_{\zeta}[v(y, t)]-\frac{1}{s^{\zeta}} \Gamma(\zeta+1) v(y, 0)+\frac{1}{s^{\zeta}} L_{\zeta}[R v(y, t)]+\frac{1}{s^{\zeta}} L_{\zeta}[N v(y, t)]-\frac{1}{s^{\zeta}} L_{\zeta}[q(y, t)]=0$. (20)

Step 3: The nth order deformation equation

$v_{n}(y, t)=\chi_{n} v_{n-1}(y, t)+h L^{-1}\left[R_{n}\left(v_{n-1}(y, t)\right)\right]$,

Where,

$$
\begin{aligned}
R_{n-1}\left[v_{n-1}(y, t)\right] & =\left[L_{\zeta}\left[v_{n-1}(y, t)\right]-\frac{1}{s^{\zeta}} \Gamma(\zeta+1) v(y, 0)+\frac{1}{s^{\zeta}} L_{\zeta}\left[R v_{n-1}(y, t)\right]\right. \\
& +\frac{1}{s^{\zeta}} L_{\zeta}\left[N v_{n-1}(y, t)\right]-\frac{1}{s^{\zeta}} L_{\zeta}[q(y, t)],
\end{aligned}
$$

Where,

$$
\chi_{n=} \begin{cases}0 & n \leq 1 \\ 1 & n>1\end{cases}
$$

\section{Application}

Consider the well-known Lorenz system in this study.

$$
\begin{aligned}
& D^{\zeta} x=\sigma(y(t)-x(t)) \\
& D^{\zeta} y=R x(t)-y(t)-x(t) z(t) \\
& D^{\zeta} z=x(t) y(t)+b z(t)
\end{aligned}
$$

where convective velocity, temperature differential between descending and ascending flows, and mean convective heat flow are proportional to $\mathrm{x}, \mathrm{y}$, and $\mathrm{z}$, and $\sigma, \mathrm{b}$, and the so called bifurcation parameter $\mathrm{R}$ are real constants. With the initial condition $\mathrm{x}(0)=\mathrm{c} 1 ; \mathrm{y}(0)=$ $\mathrm{c} 2 ; \mathrm{z}(0)=\mathrm{c} 3$.

$$
\begin{gathered}
\frac{d^{\zeta} x}{d t^{\zeta}}=\sigma(y(t)-x(t)) \\
\frac{d^{\zeta} y}{d t^{\zeta}}=R x(t)-y(t)-x(t) z(t) \\
\frac{d^{\zeta} z}{d t^{\zeta}}=x(t) y(t)+b z(t)
\end{gathered}
$$

with the initial condition $x(0)=c_{1}, y(0)=c_{2}, z(0)=c_{3}$. Apply $L_{\zeta}$ on both sides of the equation (25) 


$$
\begin{gathered}
L_{\zeta}\left[\frac{d^{\zeta} x}{d t^{\zeta}}\right]=L_{\zeta}[\sigma(y(t)-x(t))] \\
s^{\zeta} L_{\zeta}[x(t)]-\Gamma(\zeta+1) x(0)=\sigma\left[L_{\zeta}(y(t)-x(t))\right] \\
s^{\zeta} L_{\zeta}[x(t)]-\Gamma(\zeta+1) x(0)=\sigma\left[L_{\zeta}\left(y_{m-1}(t)\right)-L_{\zeta}\left(x_{m-1}(t)\right)\right]
\end{gathered}
$$

The $n$th order deformation equation for $\mathrm{x}(\mathrm{t})$ is defined as

$$
\begin{gathered}
R_{n}\left[x_{n-1}(t)\right]=\left[L_{\zeta}\left[x_{n-1}(t)\right]-\frac{1}{s^{\zeta}} \Gamma(\zeta+1) x(0)\left(1-\chi_{n}\right)-\frac{1}{s^{\zeta}} \sigma L_{\zeta}\left[y_{n-1}(t)\right]\right. \\
+\frac{1}{s^{\zeta}} \sigma L_{\zeta}\left[x_{n-1}(t)\right] \\
R_{n}\left[y_{n-1}(t)\right]=\left[L_{\zeta}\left[y_{n-1}(t)\right]-\frac{1}{s^{\zeta}} \Gamma(\zeta+1) y(0)\left(1-\chi_{n}\right)\right. \\
\quad-\frac{1}{s^{\zeta}} R L_{\zeta}\left[\left(x_{n-1}(t)\right]+\frac{1}{s^{\zeta}} L_{\zeta}\left[y_{n-1}(t)\right]\right. \\
\left.+\frac{1}{s^{\zeta}} L_{\zeta}\left[\sum_{i=0}^{m-1} x_{i}(t) z_{m-1-i}(t)\right]\right] \\
R_{n}\left[z_{n-1}(t)\right]=\left[L_{\zeta}\left[z_{n-1}(t)\right]-\frac{1}{s^{\zeta}} \Gamma(\zeta+1) z(0)\left(1-\chi_{n}\right)-\frac{1}{s^{\zeta}} L_{\zeta}\left[\sum_{i=0}^{m-1} x_{i}(t) y_{m-1-i}(t)\right]\right. \\
-\frac{1}{s^{\zeta}} b L_{\zeta}\left[z_{n-1}(t)\right]
\end{gathered}
$$

On both sides of the equation (31),(32), and (33), use the inverse fractional Laplace transform

$$
\begin{aligned}
x_{n}(t) & =\chi_{n} x_{n-1}(t)+h L^{-1}\left[R_{n}\left(x_{n-1}(t)\right]\right. \\
y_{n}(t) & =\chi_{n} y_{n-1}(t)+h L^{-1}\left[R_{n}\left(y_{n-1}(t)\right]\right. \\
z_{n}(t) & =\chi_{n} z_{n-1}(t)+h L^{-1}\left[R_{n}\left(z_{n-1}(t)\right]\right.
\end{aligned}
$$

On solving the (34) (35) (36) equations for $n=1,2,3,4, \ldots \ldots$.

$$
\begin{gathered}
x_{1}(t)=h \sigma\left(-c_{2}+c_{1}\right) \frac{t^{\zeta}}{\Gamma(\zeta+1)} \\
y_{1}(t)=-h\left(R c_{1}-c_{2}-c_{1} c_{3}\right) \frac{t^{\zeta}}{\Gamma(\zeta+1)} \\
z_{1}(t)=-h\left(c_{1} c_{2}+b c_{3}\right) \frac{t^{\zeta}}{\Gamma(\zeta+1)} \\
x_{2}(t)=\left[h \sigma\left(-c_{2}+c_{1}\right) \frac{t^{\zeta}}{\Gamma(\zeta+1)}+h^{2} \sigma\left(R c_{1}-c_{2}-c_{1} c_{3}\right) \frac{t^{2 \zeta}}{2 \Gamma^{2}(\zeta+1)}+h^{2} \sigma^{2}\left(-c_{2}\right.\right. \\
\left.\left.+c_{1}\right) \frac{t^{2 \zeta}}{2 \Gamma^{2}(\zeta+1)}\right] \\
y_{2}(t)=\left[h\left(R c_{1}-c_{2}-c_{1} c_{3}\right) \frac{t^{\zeta}}{\Gamma(\zeta+1)}+h^{2}\left(R c_{1}-c_{2} C_{1} c_{3}\right) \frac{t^{\zeta}}{\Gamma(\zeta+1)} i n\right. \\
-R h^{2} \sigma\left(-c_{2}+c_{1}\right) \frac{t^{2 \zeta}}{2 \Gamma^{2}(\zeta+1)}-h^{2}\left(R c_{1}-c_{2}-c_{1} c_{3}\right) \frac{t^{2 \zeta}}{2 \Gamma^{2}(\zeta+1)} \\
\left.-h^{2} c_{1}\left(c_{1} c_{2}+b c_{3}\right) \frac{t^{2 \zeta}}{2 \Gamma^{2}(\zeta+1)}+h^{2} \sigma\left(-c_{2}+c_{1}\right) c_{3} \frac{t^{2 \zeta}}{2 \Gamma^{2}(\zeta+1)}\right]
\end{gathered}
$$




$$
\begin{aligned}
z_{2}(t)=\left[h \left(c_{1} c_{2}\right.\right. & \left.+b c_{3}\right) \frac{t^{\zeta}}{\Gamma(\zeta+1)}+h^{2}\left(c_{1} c_{2}+b c_{3}\right) \frac{t^{\zeta}}{\Gamma(\zeta+1)}+c_{1} h^{2}\left(R c_{1}-c_{2}\right. \\
& \left.-c_{1} c_{3}\right) \frac{t^{2 \zeta}}{2 \Gamma^{2}(\zeta+1)}-h^{2} \sigma\left(-c_{2}+c_{1}\right) c_{2} \frac{t^{2 \zeta}}{2 \Gamma^{2}(\zeta+1)}+b h^{2}\left(c_{1} c_{2}\right. \\
& \left.\left.+b c_{3}\right) \frac{t^{2 \zeta}}{2 \Gamma^{2}(\zeta+1)}\right]
\end{aligned}
$$

Similarly $\mathrm{x} 3, \mathrm{x} 4, \ldots \ldots . \mathrm{y} 3, \mathrm{y} 4, \ldots \ldots \ldots$ and $\mathrm{z} 3, \mathrm{z} 4, \ldots \ldots . . \mathrm{can}$ be assessed, and a sequence of solutions can be found as follows:

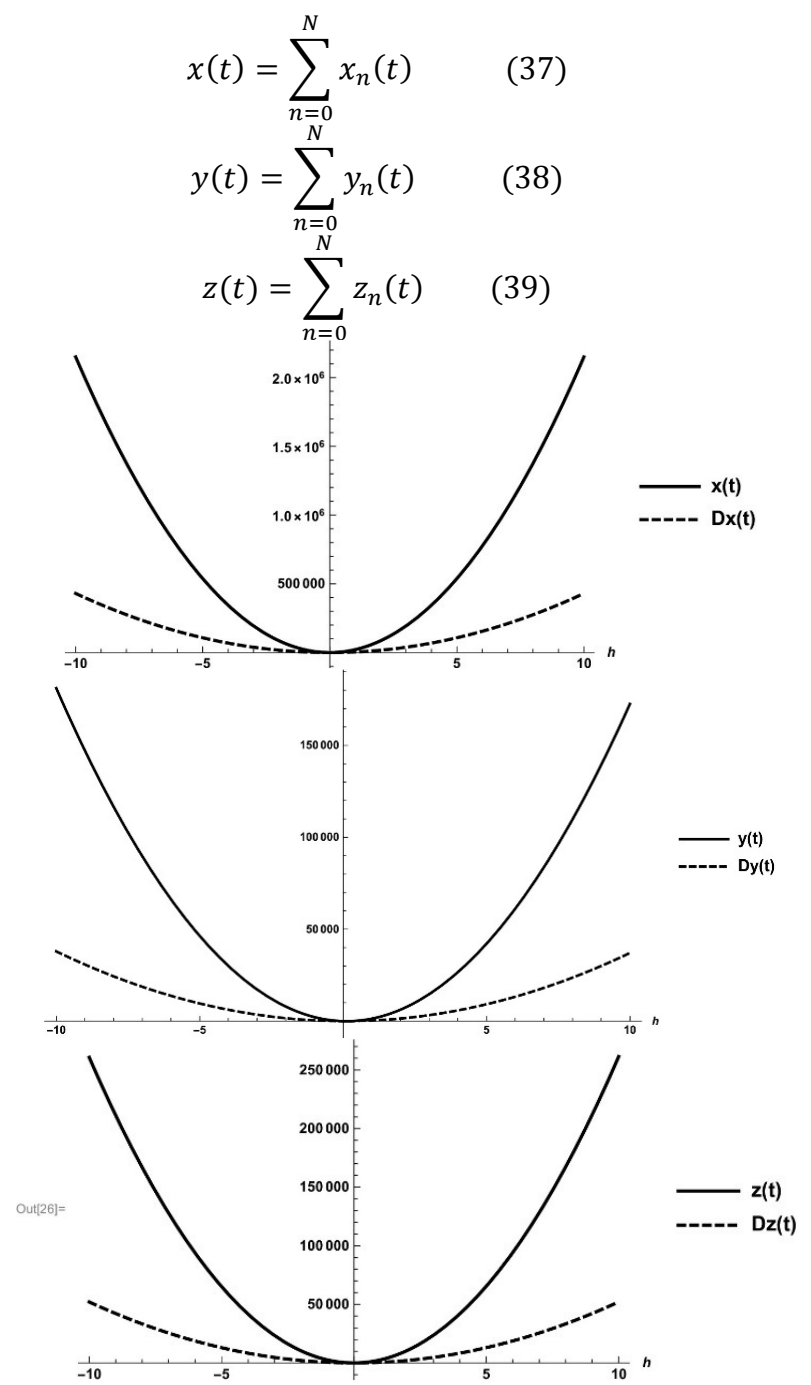

Fig. 1: The h-curve for the Lorenz system of equation $x(t)(1 a), y(t)(1 b)$ and $z(t)(1 c)$ with the convergence region for the auxiliary parameter $(\mathrm{h} \in[-1 ; 0])$. 

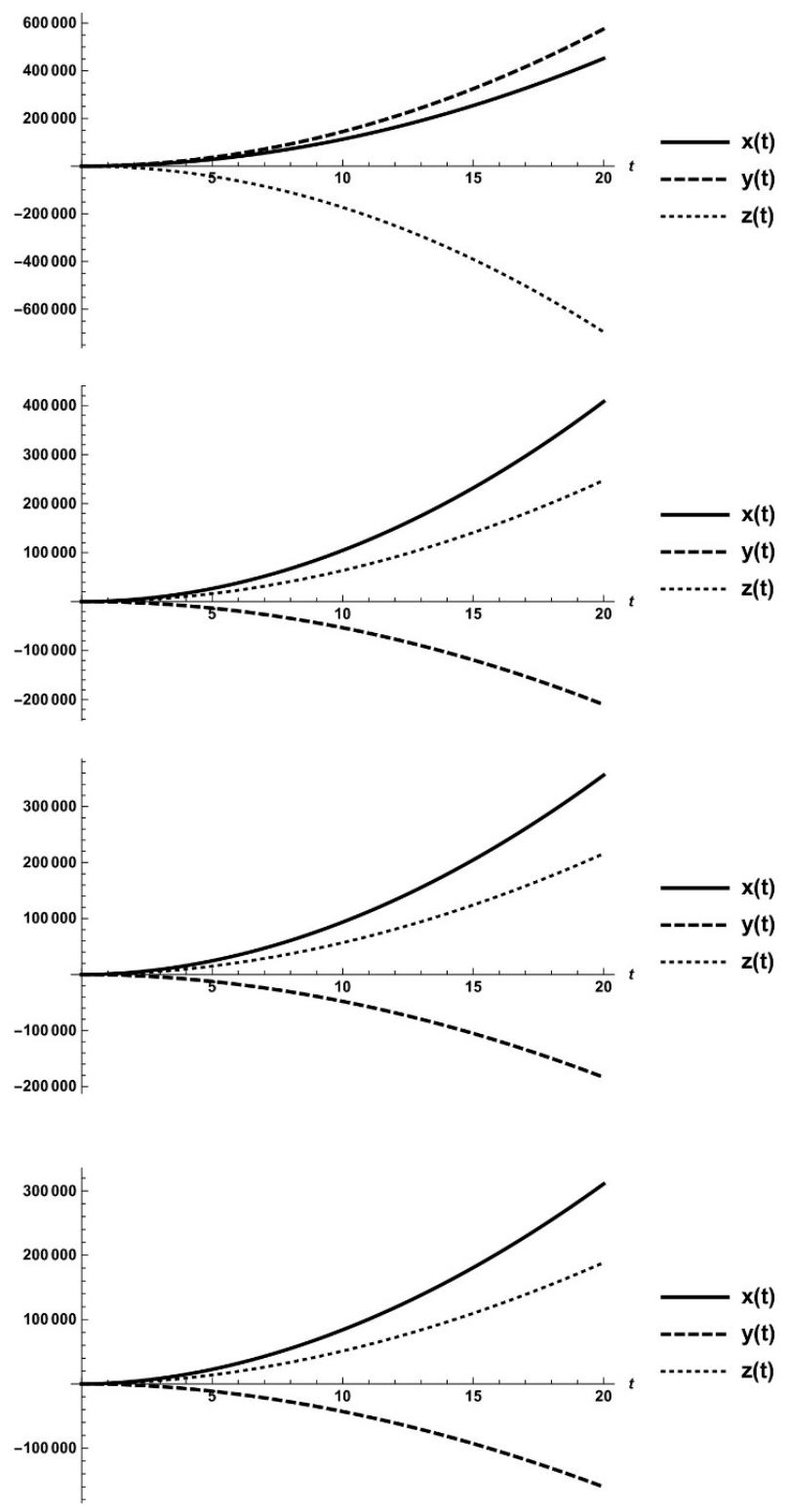

Fig. 2: The Non chaotic Solution curves for Lorenz system of equation when $\zeta$ $=1,0.98,0.96,0.94$ respectively $(2 a),(2 b),(2 c),(2 d)$. 

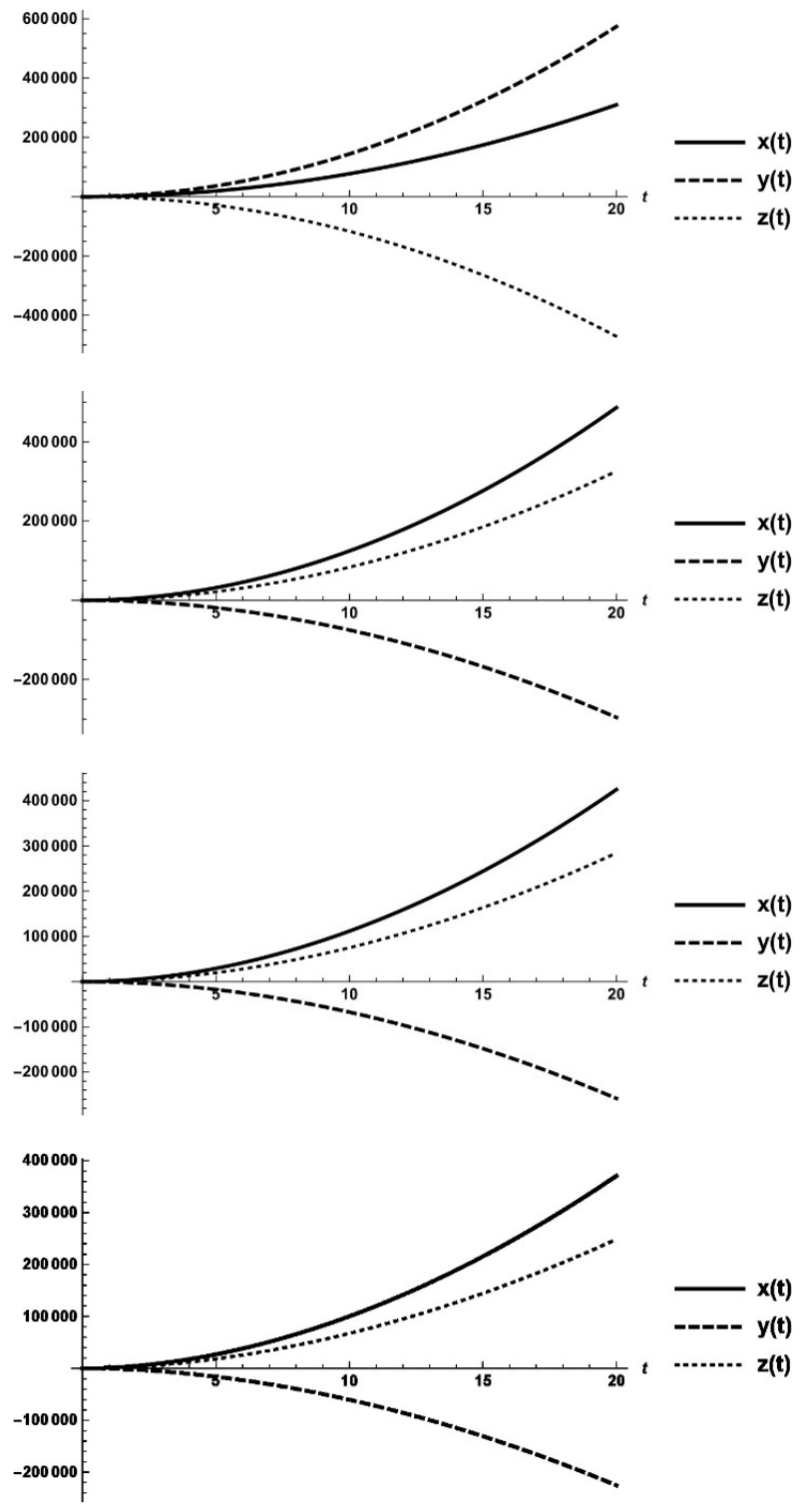

Fig. 3: The Chaotic Solution curves for Lorenz system of equation when $\zeta$ $=1,0.98,0.96,0.94$ respectively (3a), (3b), (3c), (3d)

To decide the values of $\mathrm{h}$ we plot the h-curve for the equation (37),(38) and (39) in various figures from $11-13$. From these figures, it is noted that the convergence region of $h$ lies between the range $h \in[-1,1]$. 
The solution curve of the Lorenz system are displayed in figure $7-8$ for the different values of $\zeta$. $(i . e, \zeta=0.94,0.96,0.98,1)$ and for a comparison with A.K.Alomari et al [1]. we set $\sigma$ $=10, b=-8 / 3$ we take initial conditions $x(0)=-15.8, y(0)=-17.48, z(0)=35.64$ as in [1]. Which demonstrates the excellence of the proposed Lorenz system.

TABLE I: Numerical results of Non Chaotic Solution for Lorenz system of equation when $x(t), y(t), z(t)$ for the fractional parameter $\zeta=1$ that are compared among the two methods LHAM, HAM.

\begin{tabular}{|l|l|l|l|l|l|l|}
\hline \multirow{2}{*}{$\mathrm{t}$} & \multicolumn{2}{|l|}{$\mathrm{x}(\mathrm{t})$} & $\mathrm{y}(\mathrm{t})$ & $\mathrm{z}(\mathrm{t})$ \\
\cline { 2 - 7 } & LHAM & HAM & LHAM & HAM & LHAM & HAM \\
\hline 0 & 10 & 10 & 0 & 0 & 10 & 10 \\
0.1 & 12.112 & 12.805 & 7.67927 & 7.3261 & 14.8467 & 15.2658 \\
0.2 & 38.4705 & 41.1666 & 2.53492 & 1.16086 & 34.2577 & 35.8881 \\
0.3 & 88.1468 & 94.1153 & -14.7983 & -17.8402 & 67.7334 & 71.3427 \\
0.4 & 160.717 & 171.207 & -44.0691 & -49.415 & 115.031 & 121.374 \\
0.5 & 255.899 & 272.143 & -85.1156 & -93.3944 & 175.984 & 185.808 \\
0.6 & 373.479 & 396.7 & -137.819 & -149.653 & 250.469 & 264.512 \\
0.7 & 513.288 & 544.7 & -202.084 & -218.094 & 338.385 & 357.381 \\
0.8 & 675.182 & 715.992 & -277.834 & -298.633 & 439.647 & 464.326 \\
0.9 & 859.042 & 910.449 & -365.003 & -391.203 & 554.184 & 585.271 \\
1 & 1064.76 & 1127.96 & -463.533 & -495.743 & 681.932 & -1531.32 \\
\hline
\end{tabular}

TABLE II: Numerical results of Non Chaotic Solution for Lorenz system of equation when $x(t), y(t), z(t)$ for the fractional parameter $\zeta=0.98$ that are compared among the two methods LHAM, HAM.

\begin{tabular}{|l|l|l|l|l|l|l|}
\hline \multirow{2}{*}{$\mathrm{t}$} & \multicolumn{2}{|l|}{$\mathrm{x}(\mathrm{t})$} & $\mathrm{y}(\mathrm{t})$ & $\mathrm{z}(\mathrm{t})$ \\
\cline { 2 - 7 } & LHAM & HAM & LHAM & HAM & LHAM & HAM \\
\hline 0 & -15.8 & -15.8 & -17.48 & -17.48 & 35.64 & 35.64 \\
0.1 & -6.1754 & -6.1754 & 17.7329 & 17.7329 & 36.2734 & 36.2734 \\
0.2 & 26.0584 & 26.0584 & 81.5131 & 81.5131 & 1.94479 & 1.94479 \\
0.3 & 80.9014 & 80.9014 & 173.861 & 173.861 & -67.3458 & -67.3458 \\
0.4 & 158.354 & 158.354 & 294.776 & 294.776 & -171.598 & -171.598 \\
0.5 & 258.415 & 258.415 & 444.258 & 444.258 & -310.813 & -310.813 \\
0.6 & 381.086 & 381.086 & 622.308 & 622.308 & -484.99 & -484.99 \\
0.7 & 526.365 & 526.365 & 828.925 & 828.925 & -694.128 & -694.128 \\
0.8 & 694.254 & 694.254 & 1064.11 & 1064.11 & -938.229 & -938.229 \\
0.9 & 884.753 & 884.753 & 1327.86 & 1327.86 & -1217.29 & -1217.29 \\
1 & 1097.86 & 1097.86 & 1620.18 & 1620.18 & -1531.32 & -1531.32 \\
\hline
\end{tabular}


TABLE III: Numerical results of Non Chaotic Solution for Lorenz system of equation when $x(t), y(t), z(t)$ for the fractional parameter $\zeta=0.96$ that are compared among the two methods LHAM, HAM.

\begin{tabular}{|l|l|l|l|l|l|l|}
\hline \multirow{2}{*}{$t$} & $\mathrm{x}(\mathrm{t})$ & \multicolumn{3}{|l|}{$\mathrm{y}(\mathrm{t})$} & $\mathrm{z}(\mathrm{t})$ \\
\cline { 2 - 7 } & LHAM & HAM & LHAM & HAM & LHAM & HAM \\
\hline 0 & 10 & 10 & 0 & 0 & 10 & 10 \\
0.1 & 15.295 & 14.0435 & 7.94651 & 7.17223 & 15.295 & 16.2133 \\
0.2 & 35.5032 & 45.7982 & 2.40102 & -0.529008 & 35.5032 & 38.9782 \\
0.3 & 69.6115 & 103.203 & -15.3279 & -21.71 & 69.6115 & 77.1807 \\
0.4 & 117.143 & 185.341 & -44.7415 & -55.8294 & 117.143 & 130.293 \\
0.5 & 177.777 & 291.606 & -85.5242 & -102.543 & 177.777 & 197.961 \\
0.6 & 251.276 & 421.549 & -137.446 & -161.598 & 251.276 & 279.92 \\
0.7 & 337.448 & 574.81 & -200.329 & -232.799 & 337.448 & 375.958 \\
0.8 & 436.136 & 751.095 & -274.023 & -315.982 & 436.136 & 485.899 \\
0.9 & 547.203 & 950.15 & -358.406 & -411.012 & 547.203 & 609.594 \\
1 & 670.532 & 1171.76 & -453.369 & -517.77 & 670.532 & -1531.32 \\
\hline
\end{tabular}

TABLE IV: Numerical results of Non Chaotic Solution for Lorenz system of equation when $x(t), y(t), z(t)$ for the fractional parameter $\zeta=0.94$ that are compared among the two methods LHAM, HAM.

\begin{tabular}{|l|l|l|l|l|l|l|}
\hline \multirow{2}{*}{$\mathrm{t}$} & \multicolumn{3}{|l|}{$\mathrm{x}(\mathrm{t})$} & $\mathrm{y}(\mathrm{t})$ & $\mathrm{z}(\mathrm{t})$ & \\
\cline { 2 - 7 } & LHAM & HAM & LHAM & HAM & LHAM & HAM \\
\hline 0 & 10 & 10 & 0 & 0 & 10 & 10 \\
0.1 & 12.9953 & 15.4907 & 8.20776 & 6.93477 & 15.7878 & 17.2969 \\
0.2 & 41.7532 & 50.9384 & 2.22729 & -2.45825 & 36.8311 & 42.3856 \\
0.3 & 93.3793 & 113.064 & -15.9186 & -25.9604 & 71.5903 & 83.4944 \\
0.4 & 166.644 & 200.452 & -45.4889 & -62.7352 & 119.363 & 139.808 \\
0.5 & 260.751 & 312.181 & -86.0223 & -112.258 & 179.689 & 210.79 \\
0.6 & 375.117 & 447.573 & -137.188 & -174.149 & 252.223 & 296.04 \\
0.7 & 509.28 & 606.093 & -198.727 & -248.114 & 336.697 & 395.242 \\
0.8 & 662.863 & 787.303 & -270.433 & -333.912 & 432.885 & 508.138 \\
0.9 & 835.547 & 990.831 & -352.129 & -431.342 & 540.6 & 634.504 \\
1 & 1027.06 & 1216.35 & -443.664 & -540.23 & 659.676 & 774.151 \\
\hline
\end{tabular}

TABLE V: Numerical results of Chaotic Solution for Lorenz system of equation when $x(t), y(t), z(t)$ for the fractional parameter $\zeta=1$ that are compared among the two methods LHAM, HAM.

\begin{tabular}{|l|l|l|l|l|l|l|}
\hline \multirow{2}{*}{$\mathrm{t}$} & \multicolumn{2}{|l|}{$\mathrm{x}(\mathrm{t})$} & \multicolumn{2}{l|}{$\mathrm{y}(\mathrm{t})$} & $\mathrm{z}(\mathrm{t})$ \\
\cline { 2 - 7 } & LHAM & HAM & LHAM & HAM & LHAM & HAM \\
\hline 0 & -15.8 & -15.8 & -17.48 & -17.48 & 35.64 & 35.64 \\
0.1 & -9.7304 & -9.7304 & 10.6004 & 10.6004 & 41.8903 & 41.8903 \\
0.2 & 11.8384 & 11.8384 & 67.2031 & 67.2031 & 24.4124 & 24.4124 \\
0.3 & 48.9064 & 48.9064 & 152.328 & 152.328 & -16.7937 & -16.7937
\end{tabular}




\begin{tabular}{|l|l|l|l|l|l|l|}
0.4 & 101.474 & 101.474 & 265.976 & 265.976 & -81.728 & -81.728 \\
0.5 & 169.54 & 169.54 & 408.145 & 408.145 & -170.391 & -170.391 \\
0.6 & 253.106 & 253.106 & 578.838 & 578.838 & -282.781 & -282.781 \\
0.7 & 352.17 & 352.17 & 778.052 & 778.052 & -418.9 & -418.9 \\
0.8 & 466.734 & 466.734 & 1005.79 & 1005.79 & -578.747 & -578.747 \\
0.9 & 596.798 & 596.798 & 1262.05 & 1262.05 & -762.323 & -762.323 \\
1 & 742.36 & 742.36 & 1546.83 & 1546.83 & -969.626 & 774.151 \\
\hline
\end{tabular}

TABLE VI: Numerical results of Chaotic Solution for Lorenz system of equation when $x(t), y(t), z(t)$ for the fractional parameter $\zeta=0.98$ that are compared among the two methods LHAM, HAM.

\begin{tabular}{|l|l|l|l|l|l|l|}
\hline & \multicolumn{4}{|l|}{$\mathrm{x}(\mathrm{t})$} & $\mathrm{y}(\mathrm{t})$ & $\mathrm{z}(\mathrm{t})$ \\
\cline { 2 - 7 } $\mathrm{t}$ & LHAM & HAM & LHAM & HAM & LHAM & HAM \\
\hline 0 & 10 & 10 & 0 & 0 & 10 & 10 \\
0.1 & 14.5384 & 15.364 & 9.75975 & 9.26264 & 17.2731 & 17.8248 \\
0.2 & 47.9105 & 51.1228 & 1.51521 & -0.418838 & 43.6977 & 45.8443 \\
0.3 & 109.045 & 116.156 & -23.8592 & -28.1408 & 88.6316 & 93.3838 \\
0.4 & 197.445 & 209.942 & -66.0136 & -73.5382 & 151.758 & 160.11 \\
0.5 & 312.775 & 332.13 & -124.722 & -136.375 & 232.861 & 245.794 \\
0.6 & 454.786 & 482.454 & -199.819 & -216.477 & 331.776 & 350.265 \\
0.7 & 623.275 & 660.702 & -291.171 & -313.706 & 448.373 & 473.384 \\
0.8 & 818.074 & 866.698 & -398.671 & -427.947 & 582.539 & 615.033 \\
0.9 & 1039.04 & 1100.29 & -522.226 & -559.103 & 734.181 & 775.112 \\
1 & 1286.04 & 1361.34 & -661.754 & -707.091 & 903.216 & 615.033 \\
\hline
\end{tabular}

TABLE VII: Numerical results of Chaotic Solution for Lorenz system of equation when $x(t), y(t), z(t)$ for the fractional parameter $\zeta=0.96$ that are compared among the two methods

LHAM, HAM.

\begin{tabular}{|l|l|l|l|l|l|l|}
\hline \multirow{2}{*}{$\mathrm{t}$} & \multicolumn{3}{|l|}{$\mathrm{x}(\mathrm{t})$} & $\mathrm{y}(\mathrm{t})$ & $\mathrm{z}(\mathrm{t})$ \\
\cline { 2 - 7 } & LHAM & HAM & LHAM & HAM & LHAM & HAM \\
\hline 0 & 10 & 10 & 0 & 0 & 10 & 10 \\
0.1 & 15.1429 & 16.9522 & 10.0782 & 8.98843 & 17.9128 & 19.1219 \\
0.2 & 49.9584 & 56.8052 & 1.24451 & -2.87955 & 45.4098 & 49.9851 \\
0.3 & 112.265 & 127.178 & -24.6996 & -33.6825 & 91.1899 & 101.156 \\
0.4 & 201.084 & 226.994 & -67.0605 & -82.6669 & 154.631 & 171.946 \\
0.5 & 315.77 & 355.537 & -125.398 & -149.352 & 235.317 & 261.892 \\
0.6 & 455.84 & 512.276 & -199.392 & -233.386 & 332.934 & 370.647 \\
0.7 & 620.913 & 696.787 & -288.79 & -334.492 & 447.231 & 497.934 \\
0.8 & 810.67 & 908.718 & -393.387 & -452.445 & 578.002 & 643.523 \\
0.9 & 1024.84 & 1147.77 & -513.008 & -587.053, & 725.068 & 807.216 \\
1 & 1263.2 & 1413.69 & -647.503 & -738.149 & 888.276 & 615.033 \\
\hline
\end{tabular}


TABLE VIII: Numerical results of Chaotic Solution for Lorenz system of equation when $x(t), y(t), z(t)$ for the fractional parameter $\zeta=0.94$ that are compared among the two methods

LHAM, HAM.

\begin{tabular}{|l|l|l|l|l|l|l|}
\hline \multirow{2}{*}{$\mathrm{t}$} & \multicolumn{2}{|l|}{$\mathrm{x}(\mathrm{t})$} & $\mathrm{y}(\mathrm{t})$ & $\mathrm{z}(\mathrm{t})$ \\
\cline { 2 - 7 } & LHAM & HAM & LHAM & HAM & LHAM & HAM \\
\hline 0 & 10 & 10 & 0 & 0 & 10 & 10 \\
0.1 & 15.8212 & 18.7946 & 10.3855 & 8.59375 & 18.6138 & 20.6007 \\
0.2 & 52.155 & 63.099 & 0.915056 & -5.67976 & 47.2329 & 54.5463 \\
0.3 & 115.672 & 139.126 & -25.6283 & -39.762 & 93.8828 & 109.556 \\
0.4 & 204.93 & 245.212 & -68.2145 & -92.4884 & 157.65 & 184.568 \\
0.5 & 318.993 & 380.271 & -126.201 & -163.127 & 237.93 & 278.88 \\
0.6 & 457.17 & 543.5 & -199.125 & -251.147 & 334.276 & 391.967 \\
0.7 & 618.916 & 952.054 & -286.626 & -356.137 & 446.333 & 523.417 \\
0.8 & 803.786 & 908.718 & -388.414 & -477.76 & 573.808 & 672.889 \\
0.9 & 1011.4 & 1196.42 & -504.241 & -615.733 & 716.451 & 840.091 \\
1 & 1241.43 & 1466.98 & -633.898 & -769.812 & 874.049 & 672.889 \\
\hline
\end{tabular}

The observation from Tables I, II, III, IV, V, VI, VII, VIII show that the suggested approach has a high level of agreement with HAM this study shows that LHAM is a good mathematical tool for tackling fractional Laplace Homotopy Analysis Method problems. The above tables clearly shows the changes in the Non Chaotic and chaotic situations in Lorenz system of equation with the fractional parameter $\zeta=1,0.94,0.96,0.98$.

\section{Conclusion}

In this present work continuous solution for fractional

Lorenz system of equation is obtained by Fractional Laplace Homotopy Analysis Method Via Modified Riemann-Liouville Integral. This solutions are exactly coincide with the solution of A.K.Alomari et al [1].

\section{References}

[1] A.K. Alomari A new analytic solution for fractional chaotic dynamical systems using the differential transform method (2011).

[2] A.K.Alomari,M.S.M.Noorani,R.Nazar,C.P.Li,Homotopy analysis method for solving fractional Lorenz system,commun Nonlinear Sci Number Simulat(2009).

[3] Bagyalakshmi.M,Sai Sundara Krishnan,G.Tarig Projected Differential Transform Method to solve Fractional Non linear Partial Differential Equations,Boletim da Sociedad Paranaense de Mathematica,(2018).

[4] B.K.Dutta and L.K.Arora,Fractional Elzki's Transform,PhD thesis,North Regional Institute of Science and Technology,Nirijuli-791109, Arunachal Pradesh, (2014).

[5] Fernando S.Silva,Davidson M.Moreira and Marcclo A.Moret,Conformable Laplace Transform of Fractional Differential Equation,(2018).

[6] Guy Jumarie Laplace's transform of fractional order via the Mittag Leffler function and modified RiemannLiouville derivative,Applied Mathematics Letters(2009).

[7] Guy Jumarie,Table of some basic fractional calculus formulae derived from a modified RiemannLiouville derivative for non-differentiable functions,Applied Mathematics Letters,(2008). 
[8] G.Hariharan,A.Homotopy analysis method for the nonlinear partial differential equation arising in engineering,International journal for Computational Methods in Engineering Science and mechanics,(2017).

[9] Gustavo D.Medina,Nelson R. Ojeda,Jose H.Pereira and Lui G. Romero,Fractional Laplace Transform and Fractional Calculus,International Mathematical Forum,(2008).

[10] Lorenz, E.: Deterministic nonperiodic flow1. J. Atmosph. Sci. 20, 130 (1963)

[11] M.Mossa Al-sawlha,M.S.M.Noorani On Solving the Lorenz System by Differential Transformation Method, (2008).

[12] M.S.H.Chowdhury, I.Hahim, S.Momani The multistage homotopy-perturbation method- A powerful scheme for handling the Lorenz system,(2007).

[13] Song Liang,Ranchao Wu,Liping Chen,Laplace Transform of Fractional Order Differential Equation,Electronic Of Differential Equation,(2015).

[14] Zaid M.Odibat, Cyrille Bertelle, M.A.Aziz-Alaoui, Gerard H.E.Duchamp A multi-step differential transform method and application to non-chaotic or chaotic systems (2009). 